\begin{tabular}{|c|c|c|c|}
\hline \multirow{2}{*}{$\begin{array}{r}\text { Case Reports in } \\
\text { Gastroenterology }\end{array}$} & \multicolumn{2}{|c|}{ Case Rep Gastroenterol 2016;10:63-67 } & \multirow[b]{2}{*}{$\begin{array}{l}\text { Karger } \\
\text { Open access }\end{array}$} \\
\hline & $\begin{array}{l}\text { DOI: 10.1159/000444413 } \\
\text { Publisned oninne. TVlay } 19,2016\end{array}$ & $\begin{array}{l}\text { (c) } 2016 \text { The Author(s) } \\
\text { Published by S. Karger AG, Basel } \\
\text { www.karger.com/crg }\end{array}$ & \\
\hline & $\begin{array}{l}\text { This article is licensed under } \\
\text { International License (CC BY- }- \\
\text { Usage and distribution for comn }\end{array}$ & $\begin{array}{l}\text { nons Attribution-NonCommercia } \\
\text { ger.com/Services/OpenAccessLice } \\
\text { uires written permission. }\end{array}$ & \\
\hline
\end{tabular}

\title{
Sclerosing Mesenteritis: A Rare Cause of Small Bowel Obstruction
}

\author{
Adam Graham $^{\mathrm{a}}$ Glenn Harvin ${ }^{\mathrm{b}}$ \\ ${ }^{a}$ Department of Internal Medicine, Brody School of Medicine, East Carolina University, \\ Greenville, N.C., USA; ${ }^{b}$ Division of Gastroenterology, Hepatology and Nutrition, Brody \\ School of Medicine, East Carolina University, Greenville, N.C., USA
}

\section{Keywords}

Mesenteritis $\cdot$ Small bowel obstruction $\cdot$ Bowel resection

\begin{abstract}
Sclerosing mesenteritis falls within a spectrum of primary idiopathic inflammatory and fibrotic processes that affect the mesentery. The exact etiology has not been determined, although the following associations have been noted: abdominal surgery, trauma, autoimmunity, paraneoplastic syndrome, ischemia and infection. Progression of sclerosing mesentritis can lead to bowel obstruction, a rare complication of this uncommon condition. We report a case of a 66-year-old female with abdominal pain who was noted to have a small bowel obstruction requiring laparotomy and a partial small bowel resection. The pathology of the resected tissue was consistent with sclerosing mesenteritis, a rare cause of a small bowel obstruction. Sclerosing mesenteritis has variable rates of progression, and there is no consensus regarding the optimal treatment. Physicians should consider sclerosing mesenteritis in the differential diagnosis of a small bowel obstruction.

(C) 2016 The Author(s) Published by S. Karger AG, Basel
\end{abstract}

\section{Introduction}

Sclerosing mesenteritis is a rare idiopathic inflammatory and fibrotic disorder primarily affecting the small bowel mesentery. The spectrum of this disease has included terms such as mesenteric lipodystrophy, mesenteric panniculitis, mesenteric sclerosis and retractile 
mesenteritis $[1,2]$. The mesenteric vessels and gastrointestinal lumen integrity is compromised by mass effect, which leads to the clinical findings. The most common systemic manifestations include abdominal pain, nausea, vomiting, diarrhea, weight loss and rarely ascites and small bowel obstruction. The physical examination is significant for abdominal tenderness and an abdominal mass often palpated in the left upper quadrant or epigastrium in up to $50 \%$ of patients. The mass is often deep and poorly defined. Examination findings are often nonspecific making imaging essential. The imaging modality of choice is a dual-phase abdominal computed tomography (CT). The most common finding on CT is a soft tissue mass within the small bowel mesentery. Two signs seen on CT, i.e. 'fat ring' and 'tumor pseudocapsule', are considered somewhat specific for mesenteric panniculitis. Immunohistochemical staining can also be helpful with IgG4 showing the most promise. Our case illustrates a rare case of small bowel obstruction due to sclerosing mesenteritis with immunohistochemical staining for IgG4, supporting the diagnosis of this rare cause of small bowel obstruction.

\section{Case Report}

A 66-year-old Caucasian female presented with an acute worsening of a several-year history of intermittent periumbilical abdominal pain, nausea, vomiting and diarrhea. She had an extensive workup in the past including multiple CT scans, upper endoscopies and colonoscopies without any significant findings. Her medical history is significant for mild reflux and chronic obstructive pulmonary disease. Her medications included tiotropium, esomeprazole, and as needed doxylamine succinate for sleep. On her current presentation, she reported diffuse abdominal pain and distention with an absence of flatus for 1-2 days. On examination, her abdomen was distended, tympanic to percussion and had slightly increased bowel sounds. A complete blood count was obtained with hemoglobin of $14.3 \mathrm{~g} / \mathrm{dl}$ (normal level 12.0-16.0 g/dl), a white blood cell count of $9.2 \mathrm{k} / \mu \mathrm{l}(\mathrm{nl} 4.5-11.0 \mathrm{k} / \mu \mathrm{l}$ ) and platelet count at $385 \mathrm{k} / \mu \mathrm{l}$ (normal level $150-440 \mathrm{k} / \mu \mathrm{l}$ ). An erythrocyte sedimentation rate and C-reactive protein were not collected. She underwent a CT scan showing moderate ascites and dilated loops of small bowel consistent with a small bowel obstruction. The following malignancy markers were all within the normal range: carcinoma antigen 125 was 6, carbohydrate antigen 19-9 was 8 and carcinoembryonic antigen was 0.7. A nasogastric tube was placed for decompression, and she was made NPO (nothing by mouth). General surgery was consulted, and the decision was made to operate. She underwent a laparotomy with a distal small bowel resection with a primary anastomosis. On gross inspection, the distal ileum had a thickened bowel wall and a narrowed lumen. Inflammatory cells and fibroblasts were identified on histologic review. Special immunohistologic staining was positive for IgG4. The histologic specimens are shown in figure 1a and b. Postoperatively, the patient recovered well and was discharged home. She underwent a follow-up MRI enterography of the small bowel with no remaining disease evident. She remains asymptomatic other than an occasional loose stool 1 year out from her surgery on no form of maintenance medication. She has not had a recurrence of her abdominal pain, and she has maintained a stable weight.

\section{Discussion}

Sclerosing mesenteritis is a rare inflammatory and fibrosing disorder of unknown etiology that was first described by Jura [3] in 1924. This disorder affects primarily adipose tis- 
sue of the small bowel mesentery [4] in western patients, although in Japanese patients, the disease often involves the large bowel mesentery [5]. The spectrum includes varying degrees of inflammation of the mesenteric fat, which leads to wide and variable histological features. Several possible causative factors have been noted to include abdominal surgery and trauma [6], autoimmune processes, ischemic injury, and infection, although the exact etiology is unknown [2].

Sclerosing mesenteritis often presents with nonspecific symptoms related to the mesenteric mass encasing the bowel, blood vessels and lymphatics, leading to abdominal pain, bowel obstruction, ischemia and possibly chylous ascites making the diagnosis difficult [2]. In the study by Akram et al. [1], looking at 92 cases of sclerosing mesenteritis, the most frequent presenting symptoms were abdominal pain (70\%), bloating and distention (26\%), diarrhea (25\%), and weight loss (23\%). Sclerosing mesenteritis appears to affect men twice as much as women and is usually diagnosed in the 6th or 7th decade of life [1]. Blood tests are usually normal as was the case with our patient. Occasionally, elevation of the erythrocyte sedimentation rate, neutrophilia, or anemia can be seen [7]. Colonoscopy is usually normal since the disease is extrinsic to the bowel [7], and since the disease primary involves the small bowel mesentery. There has been an association between sclerosing mesenteritis and fibrosclerotic disorders involving other organs, suggesting these conditions represent a spectrum of disorders leading to the inflammation and fibrosis of multiple organs [1].

This patient's gross pathology and histologic findings are consistent with the diagnosis of sclerosing mesenteritis. On review of the gross specimen, diffuse mesenteric thickening was seen. Fibrosis and chronic inflammation were seen microscopically (fig. 1a). Special staining for our patient was positive for IgG4-positive plasma cells (fig. 1b), which is consistent with and supports the diagnosis of sclerosing mesenteritis. Our patient's serum IgG4 level was also elevated at $103 \mathrm{mg} / \mathrm{dl}$ (normal level $4-86 \mathrm{mg} / \mathrm{dl}$ ). Some series have shown that a subset of patients with sclerosing mesenteritis have lesions that stain abundantly for IgG4 $[1,8]$, and some have proposed that sclerosing mesenteritis could belong to the class of IgG4-related sclerosing disorders [8]. In the study by Akram et al. [1], 33\% of the cases had abundant tissue infiltration of IgG4-positive plasma cells. Immunohistochemical staining has shown abundant tissue infiltration of IgG4-positive plasma cells, as well as CD3-positive T cells and CD19/CD20-positive B cells in some cases [1].

An abdominal mass is the most common finding on CT scan and is often misdiagnosed as a neoplasm. In the study by Kipfer et al. [9], a mass in the left upper quadrant was the most common, and usually the only, physical examination finding. Kipfer et al. [9] described three patterns of mesenteric involvement: type I was the most common and had diffuse mesenteric thickening; type II involved a discrete mass, and type III is the least common with multiple discrete masses. Sclerosing mesenteritis is generally considered very rare, although a retrospective study of CT images performed for various reasons suggested a prevalence of $0.6 \%$ [10].

The most common finding on CT is a soft tissue mass within the small bowel mesentery. Two signs seen on CT 'fat ring' and 'tumoral pseudocapsule' are considered somewhat specific for mesenteric panniculitis [5]. The 'fat ring' sign is due to preservation of the fat nearest the mesenteric vessels [11], while the 'tumoral pseudocapsule' represents separation of the uninvolved mesentery from the inflamed fat by a band of tissue [12]. Some have reported an increased association with malignancies including lymphomas, urogenital or gastrointenstinal adenocarcinomas [10], although this is unclear [2]. In the study by Daskalogiannaki et al. [10], $69 \%$ of patients with mesenteric panniculitis diagnosed by CT scan had a coexisting malignancy. 
The differential diagnosis includes other diseases that can affect the mesentery including lymphomas, desmoid tumors, carcinoid tumors, carcinomatosis, amyloidosis, and mesenteric fibromatosis. Definitive diagnosis requires a surgical excision, although the diagnosis is often made based upon the findings on CT scan. It is believed to have a benign, stable or slowly progressive course in the majority of cases, although there are cases of rapidly fatal disease [13]. There is no consensus regarding the optimal treatment, and numerous agents such as steroids, colchicine, azathioprine, tamoxifen and others have been used with varying success [7]. Surgery is usually indicated for a small bowel obstruction, as was the case in our patient. Many of the treatment regimens are anecdotal due to the rarity of the disease, although one suggested regimen for patients with nonobstructive symptoms is a combination of tamoxifen with a prednisone taper over 3 months [5].

Sclerosing mesenteritis is a rare cause of small bowel obstruction with varying degrees of progression. Due to the nonspecific symptoms, diagnosis is often challenging. CT scans are often suggestive but not necessarily diagnostic. Various treatment regimens have been used, although often anecdotal. Clinicians must maintain a high level of suspicion for the disease, and this must be included in the differential diagnosis of a case of small bowel obstruction.

\section{Statement of Ethics}

Informed consent was obtained from the patient.

\section{Disclosure Statement}

Neither author received any financial support for the manuscript or has any financial or other conflict of interest.

\section{References}

1 Akram S, Pardi DS, Schaffner JA, Smyrk TC: Sclerosing mesenteritis: clinical features, treatment, and outcome in ninety-two patients. Clin Gastroenterol Hepatol 2007;5:589-596; quiz 523-524.

-2 Vlachos K, Archontovasilis F, Falidas E, Mathioulakis S, Konstandoudakis S, Villias C: Sclerosing mesenteritis: diverse clinical presentations and dissimilar treatment options. A case series and review of the literature. Int Arch Med 2011;4:17.

3 Jura V: Mesenterite e sclerosante. Policlinico 1924;31:575-581.

4 McCrystal DJ, O'Loughlin BS, Samaratunga H: Mesenteric panniculitis: a mimic of malignancy. Aust NZ J Surg 1998;68:237-239.

5 Endo K, Moroi R, Sugimura M, et al: Refractory sclerosing mesenteritis involving the small intestinal mesentery: a case report and literature review. Intern Med 2014;53:1419-1427.

-6 Emory TS, Monihan JM, Carr NJ, Sobin LH: Sclerosing mesenteritis, mesenteric panniculitis and mesenteric lipodystrophy: a single entity? Am J Surg Pathol 1997;21:392-398.

7 Issa I, Baydoun H: Mesenteric panniculitis: various presentations and treatment regimens. World Gastroenterol 2009;15:3827-3830.

8 Chen TS, Montgomery EA: Are tumefactive lesions classified as sclerosing mesenteritis a subset of IgG4related sclerosing disorders? J Clin Pathol 2008;61:1093-1097.

-9 Kipfer RE, Moertel CG, Dahlin DC: Mesenteric lipodystrophy. Ann Intern Med 1974;80:582-588.

10 Daskalogiannaki M, Voloudaki A, Prassopoulos P, et al: CT evaluation of mesenteric panniculitis: prevalence and associated diseases. AJR Am J Roentgenol 2000;174:427-431.

11 Valls C: Fat-ring sign in sclerosing mesenteritis. AJR Am J Roentgenol 2000;174:259-260.

12 Sabate JM, Torrubia S, Maideu J, Franquet T, Monill JM, Perez C: Sclerosing mesenteritis: imaging findings in 17 patients. AJR Am J Roentgenol 1999;172:625-629. 


\section{Case Reports in \\ Case Rep Gastroenterol 2016;10:63-67 \\ Gastroenterology \\ (C) 2016 The Author(s). Published by S. Karger AG, Basel www.karger.com/crg \\ Graham and Harvin: Sclerosing Mesenteritis: A Rare Cause of Small Bowel Obstruction}

13 Mazure R, Fernandez Marty P, Niveloni S, et al: Successful treatment of retractile mesenteritis with oral progesterone. Gastroenterology 1998;114:1313-1317.

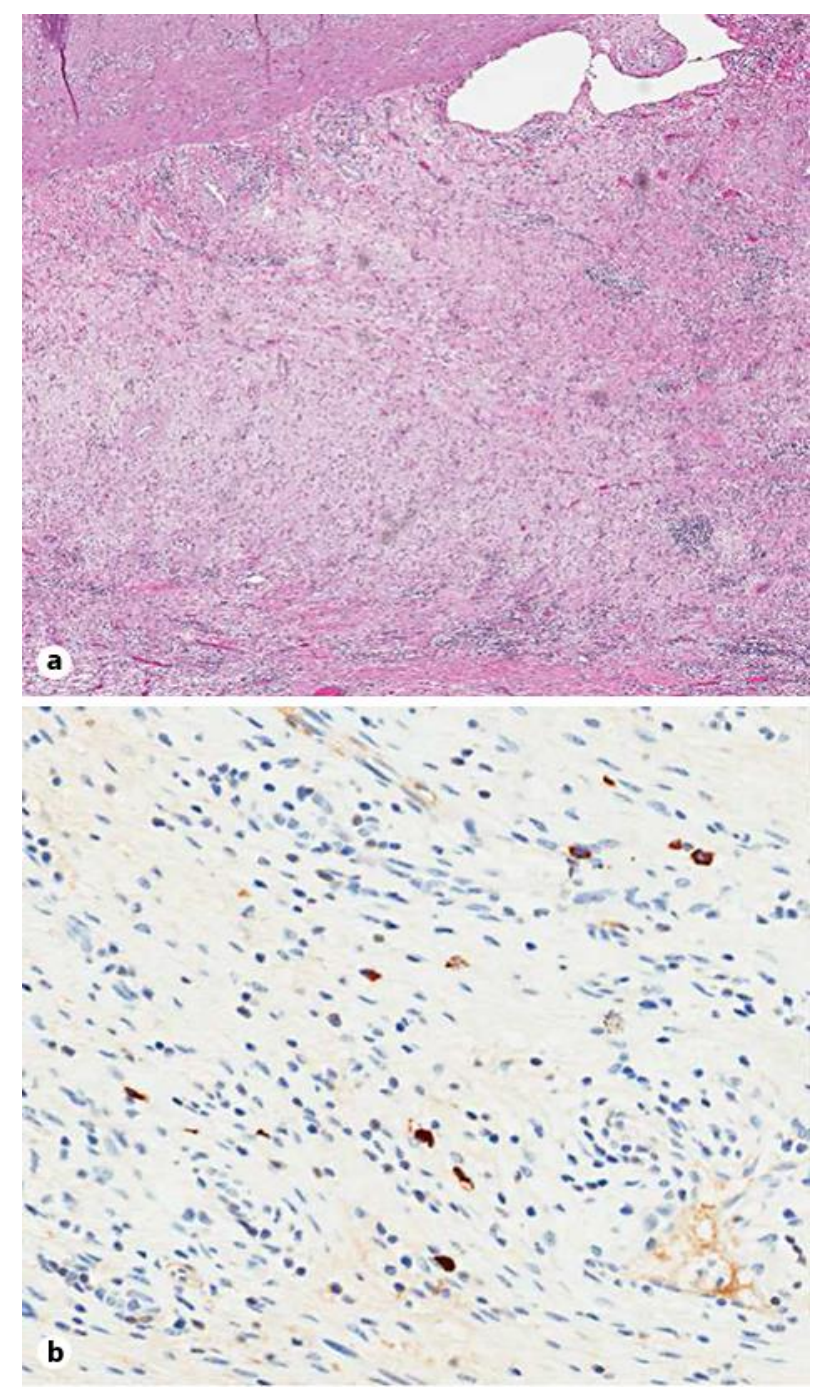

Fig. 1. a Hematoxylin and eosin stain illustrating fibrosis and chronic inflammation. $\times 40$. $\mathbf{b}$ Tissue staining positive for IgG4 plasma cells. $\times 100$. 